\title{
Dosimetric Comparison between High Dose Rate Brachytherapy Boost and Volumetric Arc Therapy Boost in Locally Advanced Cancer Cervix
}

\author{
Hanady Hegazy ${ }^{1} \quad$ Neamat Hegazy ${ }^{1} \quad$ Maher Soliman $^{1} \quad$ Amr Elsaid ${ }^{1}$ \\ ${ }^{1}$ Department of Clinical Oncology and Nuclear Medicine, Alexandria \\ Address for correspondence Hanady Hegazy, PhD , Department \\ Clinical Oncology Department, Alexandria, Egypt \\ of Clinical Oncology and Nuclear Medicine, Alexandria \\ Clinical Oncology Department, Alexandria 21131, Egypt \\ (e-mail: hanajodyan@yahoo.com).
}

\begin{abstract}
Concurrent chemoradiotherapy is considered the standard treatment for the locally advanced cancer cervix (LACC). Radiotherapy is commonly administered by a three-dimensional conformal radiotherapy (3DCRT) approach followed by brachytherapy (BT). High dose rate (HDR) BT is commonly administered; however, several drawbacks exist including invasive technique, pain, requirement of anesthesia, and operative risks. We assessed the dosimetric difference between the HDR BT and the volumetric modulated arc therapy (VMAT) boost in those patients. Ten patients were selected retrospectively with LACC and all received whole pelvis radiotherapy followed by BT boost of 7 Gy in three fractions. The computed tomography (CT) image was transferred to the Varian system for the VMAT plan while the one with the applicator was transferred to the Sagi planning system and the high-risk clinical target volume (HR-CTV), bladder, rectum, sigmoid, and small bowel were delineate with a margin of $5 \mathrm{~mm}$ were added to the CTV to create the planning target volume (PTV). The D90 for

Keywords

- LACC

- HDR BT

- VMAT

- HRCTV the PTV in VMAT boost was lower than received by the HR-CTV in the BT boost. Mean volume of the PTV was higher than that of the HR-CTV. The D2cc was higher in VMAT for bladder, sigmoid, and rectum while the D2cc for the small bowel in BT was higher compared with the VMAT. The VMAT is an option that exists for patients who refuse BT or cannot tolerate it, or in case of nonavailability of BT or a nonworking machine.
\end{abstract}

\section{Introduction}

Globally, cancer cervix is still one of the most common cancers in females, being the fourth most common after breast, colorectal, and lung cancer. ${ }^{1}$ In Egypt, cancer of cervix is the 13th most frequent cancer among females and the 10th most frequent cancer among females aged between 15 and 44 years. $^{2}$ Concomitant chemoradiation is considered the standard treatment of locally advanced cancer cervix (LACC). The radiotherapy treatment consists of external beam

DOI https://doi.org/ $10.1055 / \mathrm{s}-0041-1729480$ ISSN 2454-6798 radiotherapy (EBRT) and brachytherapy (BT) boost. Although using high dose rate (HDR) BT provides a unique pear-shaped dose distribution with steep dose gradients, it also contains certain limitations including being an invasive technique, require preplanning before each session, and the risk of anesthesia. ${ }^{3}$ Volumetric modulated arc therapy (VMAT) was first introduced in 2007 and described as a novel radiation technique that allowed the simultaneous variation of three parameters during treatment delivery, that is, gantry rotation speed, treatment aperture shape via movement of multileaf

@2021. Spring Hope Cancer Foundation \& Young Oncologist Group of Asia.

This is an open access article published by Thieme under the terms of the Creative Commons Attribution-NonDerivative-NonCommercial-License, permitting copying and reproduction so long as the original work is given appropriate credit. Contents may not be used for commercial purposes, or adapted, remixed, transformed or built upon. (https://creativecommons.org/licenses/by-nc-nd/4.0/).

Thieme Medical and Scientific Publishers Pvt. Ltd. A-12, 2nd Floor, Sector 2, Noida-201301 UP, India 
collimator (MLC) leaves, and dose rate. ${ }^{4}$ VMAT showed a great potential for producing highly conformal doses to treatment volumes while sparing the organs at risk (OARs). ${ }^{5}$ On the other hand, it was unable to deliver an increased homogenous dose to the planning target volume (PTV) while appropriately sparing the OARs. VMAT may be potentially an alternative option for duplicating traditional BT dose distributions for patients in need of BT who are unable to undergo this treatment modality. ${ }^{5}$ Toxicity to OARs should always be considered when using any EBRTX technique to treat those who are unable to receive BT treatment with the gastrointestinal tract's toxicity being the main recorded toxicity. ${ }^{3}$ Also the considerations for target motion and delineation made the VMAT technique limited compared with the traditional BT technique. ${ }^{6}$ So in our patients, we started a dosimetric comparison between the BT boost and the EBRT with VMAT boost.

\section{Methods and Materials}

Ten patients were selected with LACC who achieved response after EBRT. The patients received whole pelvis three-dimensional (3D) conformal EBRT ( 45 Gy in 25 fractions) with assumption that OARs received 100\% of the dose. A boost was given by intracavitary computed tomography (CT)-guided HDR BT (7 Gy/3f). High-risk clinical target volume (HR-CTV), bladder, rectum, and small bowel were delineated. Planning was done using Sagi planning system and was manually optimized with respect to organ dose limits.

\section{The Brachytherapy Boost}

HDR BT (cobalt 60) was used with intracavitary applicator.

- It was done under spinal or epidural anesthesia.

- Applicators-tandem 40/60, ring (30-35-25).

- Examination was done under anesthesia and dilatation of the cervix then insertion of the applicators was done.

- A CT scan was done to ensure good application, then images were transferred to the CT planning (Sagi planning system) where reconstruction was done.

- Contouring of the HR-CTV, which is defined by GEC-ESTRO, ${ }^{7}$ as the area of gross residual disease at the time of BT. It includes the gross disease at the time of implant, the entire cervix, and any areas clinically suspicious for residual disease. Organs at risk include rectum, urinary bladder, and small bowel.

- Then a plan was generated to achieve our goal:

- A dose to the $90 \%$ of the target of $7 \mathrm{~Gy} / \mathrm{f}$ in three fractions, (D90 = $7 \mathrm{~Gy}$ ).

- A dose to D2cc rectum $<4 \mathrm{~Gy} / \mathrm{f}$, sigmoid $<4 \mathrm{~Gy} /$ f, and urinary bladder $<5 \mathrm{~Gy} / \mathrm{f}$ in each fraction.

All the patients had CT with contrast before the insertion of the intracavitory applicator and the CDs were transferred to the Varian planning system for generating the VMAT plans. A margin of $5 \mathrm{~mm}$ was added to the CTV to create the target planning target volume (PTV). The inversely planned VMAT was challenged to deliver the highest possible doses to PTVs while respecting D2cc limits from BT, assuming the same fractionation (7 Gy in three fractions).
The VMAT boost was created with the following steps: Target volume delineation and organs at risk delineation were done taking in consideration the following:

- Two different planning systems in two separate places.

- The attempts to unify the volumes between the VMATS plans and the BT plans.

- HR-CTV in the BT boost was delineated in the VMAT boost as CTV then $5 \mathrm{~mm}$ around as a PTV.

- Two full arcs were used with collimator angel (90-0).

- Energy used 6 MV.

- Dose-volume histogram was used to assess the points of comparisons between the two plans.

- HDR radiobiological dose equivalent worksheets available on the American Brachytherapy Society (ABS) online were used for dosimetric calculations.

For the VMAT boost, the same fractionation was assumed as for the BT-7 Gy $\times 3$ fractions to cover the PTV after 45 Gy of the EBRT. Inversely planned VMAT was challenged to deliver the highest possible doses to the PTV while maintaining the same D2cc limits for bladder, rectum, and sigmoid. Available DVH information from BT was used as input parameters during inverse planning with achieving the dose constrains to the OARs illustrated in - Table 1.

The primary target parameter of interest was the D90. For VMAT the dose was to be delivered to the PTV structures and not to the CTV as in BT.

If D90 values of BT could not be reproduced with VMAT, it was aimed to achieve a D90 of 7 Gy for the PTV.

For treatment plan evaluation, D90 for PTV achieved with VMAT were compared with the HRCTV values from advanced BT. Besides, D2cc which was used for treatment planning for the OAR bladder, rectum, and sigmoid were evaluated.

All dosimetric data of different plans were compared with a paired two-tailed student's $t$-test, and statistical significance was assumed at $p<0.05$.

\section{Results}

When VMAT was limited to D2cc from BT, the D90 for the PTV was lower than that received by the HR CTV in the BT boost (6.3 Gy vs. $6.9 \mathrm{~Gy}, p$-value 0.037). Mean volume of the PTV in VMAT is higher than that of HR CTV in the BT $\left(89 \mathrm{~cm}^{3} \mathrm{vs}\right.$. $\left.41.7 \mathrm{~cm}^{3}\right)$ (-Table 2).

The dose to the OARs was comparable. D2cc was higher in VMAT for bladder and rectum while D2cc for sigmoid and small bowel in BT was higher compared with the VMAT. HR-CTV in the BT $\left(89 \mathrm{~cm}^{3}\right.$ vs. $\left.41.7 \mathrm{~cm}^{3}\right)(-$ Table 3$)$.

Table 1 Dose constrains to the organs at risk (OARs) in the VMAT plans ${ }^{8}$

\begin{tabular}{|l|l|}
\hline OARs & Dose constrains \\
\hline Rectum & V50 $<50 \%$ \\
& V60 $<15 \%$ \\
\hline Bladder & V40 $<50 \%$ \\
& V65 $<50 \%$ \\
\hline Small bowel & V50 $<5 \%$ \\
\hline
\end{tabular}


High Dose Rate Brachytherapy and Volumetric Arc Therapy in Locally Advanced Cervical Cancer Hegazy et al. 87

Table 2 Comparison between brachytherapy and VMAT according to the HR-CTV/PTV integral dose and parameters

\begin{tabular}{|c|c|c|c|c|}
\hline Parameters & Brachytherapy & VMAT & Z & $p$-Value \\
\hline \multicolumn{5}{|c|}{$\begin{array}{l}\text { Integral dose to HR-CTV/PTV } \\
\text { (mean dose } \times \text { volume) }\end{array}$} \\
\hline Min-max & $50.3-588.0$ & $468.6-1027.5$ & \multirow[t]{3}{*}{$2.803^{\mathrm{a}}$} & \multirow[t]{3}{*}{$0.005^{\mathrm{a}}$} \\
\hline Mean \pm SD & $191.6 \pm 161.6$ & $671.0 \pm 185.6$ & & \\
\hline Median (IQR) & $119.8(101.8-255.6)$ & $653.2(502.3-752.0)$ & & \\
\hline \multicolumn{5}{|l|}{ Mean dose } \\
\hline Min-Max & $3.4-6.8$ & $7.1-7.9$ & \multirow[t]{3}{*}{$2.810^{\mathrm{a}}$} & \multirow[t]{3}{*}{$0.005^{\mathrm{a}}$} \\
\hline Mean \pm SD & $5.1 \pm 1.2$ & $7.5 \pm 0.3$ & & \\
\hline Median (IQR) & $5.4(3.9-6.0)$ & $7.6(7.4-7.7)$ & & \\
\hline \multicolumn{5}{|c|}{ HR-CTV/PTV volume $\mathrm{cm}^{3}$} \\
\hline Min-Max & 7.4-149.6 & $65.0-137.0$ & \multirow[t]{3}{*}{$2.497^{a}$} & \multirow[t]{3}{*}{$0.013^{\mathrm{a}}$} \\
\hline Mean \pm SD & $41.7 \pm 41.8$ & $89.0 \pm 23.6$ & & \\
\hline Median (IQR) & $23.3(19.8-53.9)$ & 85.8 (66.7-99.1) & & \\
\hline \multicolumn{5}{|l|}{ HR-CTV/PTV D90 } \\
\hline Min-Max & $5.7-8.1$ & $6.2-6.4$ & \multirow[t]{3}{*}{$2.090^{\mathrm{a}}$} & \multirow[t]{3}{*}{$0.037^{a}$} \\
\hline Mean \pm SD & $6.9 \pm 0.80$ & $6.3 \pm 0.1$ & & \\
\hline Median (IQR) & $7.0(6.6-7.2)$ & $6.3(6.3-6.3)$ & & \\
\hline
\end{tabular}

Abbreviations: HR-CTV, high-risk clinical target volume; IQR, interquartile range PTV, planning target volume; SD, standard deviation; VMAT, volumetric modulated arc therapy.

astatistically significant at $p \leq 0.05$.

Table 3 Comparison between BT and VMAT according to the D2cc of the OARs

\begin{tabular}{|c|c|c|c|c|}
\hline OAR D2cC & BT & VMAT & Z & $p$-Value \\
\hline Rectum & & & \multirow[t]{5}{*}{$2.599^{\mathrm{a}}$} & \multirow[t]{5}{*}{$0.009^{a}$} \\
\hline Min-max & $3.1-4.7$ & $3.8-7.0$ & & \\
\hline Mean \pm SD & $3.9 \pm 0.5$ & $5.5 \pm 1.0$ & & \\
\hline Median (IQR) & $4.1(3.3-4.3)$ & $5.3(4.9-6.3)$ & & \\
\hline \multicolumn{3}{|l|}{ Bladder } & & \\
\hline Min-max & $3.2-5.9$ & $4.3-6.7$ & \multirow[t]{3}{*}{$2.803^{\mathrm{a}}$} & \multirow[t]{3}{*}{$0.005^{\mathrm{a}}$} \\
\hline Mean \pm SD & $4.3 \pm 0.9$ & $5.6 \pm 0.9$ & & \\
\hline Median (IQR) & $4.0(3.5-5.3)$ & $5.7(4.8-6.5)$ & & \\
\hline \multicolumn{3}{|l|}{ Sigmoid } & \multirow[t]{4}{*}{$2.803^{a}$} & \multirow[t]{4}{*}{$0.005^{a}$} \\
\hline Min-max & $0.68-4.33$ & $0.20-1.27$ & & \\
\hline Mean \pm SD & $3.19 \pm 1.18$ & $0.51 \pm 0.36$ & & \\
\hline Median (IQR) & $3.44(2.5-4.2)$ & $0.38(0.25-0.65)$ & & \\
\hline \multicolumn{3}{|l|}{ Small bowel } & \multirow[t]{4}{*}{1.784} & \multirow[t]{4}{*}{0.074} \\
\hline Min-max & $2.0-7.0$ & $0.20-4.8$ & & \\
\hline Mean \pm SD & $4.1 \pm 1.7$ & $1.94 \pm 1.90$ & & \\
\hline Median (IQR) & $3.7(2.5-5.3)$ & $1.3(0.35-4.30)$ & & \\
\hline
\end{tabular}

Abbreviations: BT, brachytherapy; IQR, interquartile range; OAR, organ at risk; SD, standard deviation; VMAT, volumetric modulated arc therapy. Notes: Z-Wilcoxon signed ranks test.

p-Value for comparing between BT and VMAT.

aStatistically significant at $p \leq 0.05$.

\section{Discussion}

Considering that all patients received 3D CRT whole pelvis radiotherapy in a dose of $45 \mathrm{~Gy}$ in 25 fractions and taking in consideration that the OARs received the full dose. The BT boost received was $7 \mathrm{~Gy}$ in three fractions and the same dose with its calculated biological equivalent dose was used for the VMAT boost under study. When VMAT was limited to D2cc from BT, the D90 for the PTV was lower significantly than that in the BT boost to the HR-CTV (6.3 Gy vs. $6.8 \mathrm{~Gy}$ with $p$-value 0.037 ) and variation of the dose with hot areas in BT compared with more homogenous dose in the VMAT 
(5.7-8.1 Gy in BT vs. 6.2-6.4 Gy in the VMAT). This coincides with the findings of the study done by Sergeevna ${ }^{9} \mathrm{et}$ al where the BT boost resulted in irradiation of significant target volumes by doses significantly higher than the prescribed dose while the VMAT boost significantly improved this situation. Mean volume of the PTV in VMAT is higher than that of HR-CTV in the BT. The dose to OARs was comparable where the D2cc was higher in VMAT for bladder and rectum (5.6 Gy and 5.5 Gy vs. 4.3 Gy and 3.9 Gy for bladder and rectum, respectively) while D2cc for the sigmoid and small bowel in BT were higher compared with the VMAT (3.19 Gy and 4.1 Gy vs. 0.51 Gy and 1.94 Gy for sigmoid and small bowel, respectively). This coincides with what was found in the trial published by Sethi et al ${ }^{6}$ where VMAT had a potential for improved sparing of most normal tissues compared with BT boost. The mean dose to the bladder, rectum, and small bowel were lower in the VMAT plan compared with the BT boost with excellent PTV coverage. Also in comparison with the findings of the study done by Wali et al, ${ }^{10}$ the VMAT achieved significant dose reduction of rectum, bladder, and sigmoid, as well as superior homogeneous target coverage compared with BT plan. On the other hand, VMAT delivered more radiation exposures to small bowel.

It is important to illustrate that the radiation dose and coverage depend on target and OAR contouring and motion, and this is definitely affected by the placement of the applicator and Foley catheter. In our study the BT boost parameters were calculated while the intracavitory applicator and packing were in place while the VMAT boost calculation were done before the applicator or packing were in place and this caused different position and volumes to be irradiated, although all attempts were made to unify the volumes irradiated between the two boosts. Also the addition of internal target volume (ITV) for the VMAT boost to count for the possible mobility and varying position of the OARs will possibly affect the dose to the OARs. Besides, the small number of patients involved in the study and the nonavailability of one working software that can compare the targets and OARs between the BT and VMAT on spot.

The aim of the VMAT boost application is not to replace the BT boost but to find an alternative accessible option with comparable results. The main advantages of the VMAT boost are to facilitate the treatment procedure, reduce patient discomfort resulting from the BT applicator, reduce the time of irradiation session, and avoid the anesthesia with all its possible complications.

\section{Conclusion}

The VMAT had comparable target coverage with more homogenous dose and avoidance of hot areas in the target and the potential for improved sparing of most normal tissues compared with BT boost. It is an option that exists for patients who refuse BT or cannot tolerate it or in case of nonavailability of BT or nonworking of machine. Further dosimetric analysis is needed on larger number of patients and ensure the availability of one software that involves both BT and VMAT on the spot. Besides further application of the VMAT beyond the level of dosimetric study to compare the side effects result from the VMAT compared with the BT boost.

\section{Recommendation}

It is recommended to further apply the VMAT boost on larger number of patients and compare it not only to the intracavitory BT but also interstitial BT that can cover the target properly.

\section{Conflict of Interest \\ None declared.}

\section{Acknowledgments}

The authors thank the radiation oncology physicists Ahmed Alderiny and Fady Samir for their dedicated work and support.

\section{References}

1 Ferlay J, Soerjomataram I, Dikshit R, et al. Cancer incidence and mortality worldwide: sources, methods and major patterns in GLOBOCAN 2012. Int J Cancer 2015;136(5):E359-E386

2 Bruni L, Barrionuevo-Rosas L, Albero G, Serrano B, Mena M, Gómez D, et al. ICO information centre on HPV and cancer (HPV information centre). Human papillomavirus and related diseases in the world Summary Report 2015; 4(08).

3 Otto K. Volumetric modulated arc therapy: IMRT in a single gantry arc. Med Phys 2008;35(1):310-317

4 Merrow C, deBoer S, Podgorsak MB. VMAT for the treatment of gynecologic malignancies for patients unable to receive HDR brachytherapy. J Appl Clin Med Phys 2014;4:210-217

5 Thomadsen B, Lin SW, Laemmrich P, et al. Analysis of treatment delivery errors in brachytherapy using formal risk analysis techniques. Int J Radiat Oncol Biol Phys 2003;57(5):1492-1508

6 Sethi RA, Jozsef G, Grew D, et al. Is there a role for an external beam boost in cervical cancer radiotherapy? Front Oncol 2013;3:3

7 Dimopoulos JCA, Petrow P, Tanderup K. Recommendations from Gynaecological (GYN) GEC-ESTRO Working Group (IV): basic principles and parameters for MR imaging within the frame of image based adaptive cervix cancer brachytherapy. Radiother Oncol 2012;7:55-62

8 Wu Y, Zhu B, Han J, et al. A comparative dosimetric study of cervical cancer patients with para-aortic lymph node metastasis treated with volumetric modulated arc therapy $v s$. 9-field intensity-modulated radiation therapy. Ann Transl Med 2019;7(22):675

9 Sukhikh ES, Sukhikh LG, Lushnikova PA, Tatarchenko MA, Abdelrahman AR. Dosimetric and radiobiological comparison of simultaneous integrated boost and sequential boost of locally advanced cervical cancer. Phys Med 2020;73:83-88

10 Wali L, Helal A, Darwesh R, Attar M. A dosimetric comparison of volumetric modulated arc therapy (VMAT) and high dose ratsse (HDR) brachytherapy in localized cervical cancer radiotherapy. J XRay Sci Technol 2019;27(3):473-483 